\title{
Temporal and Spatial Characteristics and Influencing Factors of Public Space Vitality in Historical Blocks-Taking Songcheng First Road in Zhaoqing as an Example
}

\author{
Yuanyuan Zhang ${ }^{1, *}$, Ziqian Chen ${ }^{1}$ and Zehui Chen ${ }^{2}$
}

\author{
${ }^{1}$ Department of Resources and the Urban Planning, Guangzhou Xinhua University, Guangzhou 510520, China \\ ${ }^{2}$ Faculty of Innovation and Design, City University of Macau, 999078, Macao, China \\ *Corresponding author. Email: zsu.zyy@163.com
}

\begin{abstract}
This study aims to construct a perfect evaluation index system for the public space vitality in historical blocks and explore the influence of different factors on space vitality. Taking the ancient city wall historical block of Songcheng first road in Zhaoqing as the research object, the temporal and spatial change characteristics of crowd behavior in historical blocks were firstly analyzed by questionnaire and network big data. Then, the vitality evaluation index system in historical blocks was analyzed combined with literature review and survey data, and the weight value of impact factors was calculated by exploratory factor analysis and AHP. Finally, the strategy to improve the historical blocks vitality was put forward based on important factors and research area. The results showed that the block location at the spatial scale level, block safety and greening coverage at the aesthetic attribute level, block sanitation, architectural characteristics and visual effects were the strong correlation factors affecting the block vitality. The research also found that people have subjective intentional cognition on overall situation of the block, and thereby more attention should be paid to maintaining spatial quality and cultural characteristics in the block construction in the future. This study added the corresponding indicators that could reflect the subjective feelings of the population from the perspective of individual psychology, which is of certain value to enrich the research on block space vitality.

Keywords: Historical blocks; Vitality evaluation; Temporal and spatial characteristics; Exploratory factor

analysis

\section{INTRODUCTION}

As a significant material space carrier for the city to display its cultural heritage, the historical block, in which the public space is an important part affecting the revival of the block [1]. In recent years, the public space vitality in many historical blocks has declined sharply or the distribution of internal space vitality has been seriously uneven [2]. Therefore, it is of great value to explore the factors affecting its vitality.

"Block vitality" was first proposed by Jane Jacobs. European and American countries have studied block vitality earlier, and the research entry points are abundant, including visual aesthetics, crowd spatial communication behavior needs and characteristics, individual perception, and the sense of belonging in public space [3-6]. The research is mainly qualitative analysis. The research on block vitality in China started relatively late, mainly focusing on three aspects: evaluation and measurement on block vitality, impact factor analysis and vitality promotion

tends to material facilities in the early stage [7]. In recent years, the evaluation criteria tend to be diversified, including the construction of evaluation indicators from the aspects of material, use and function [8]. The impact factors can be divided into material spatial and immaterial spatial elements. Material spatial elements mainly include spatial characteristics, facility status, land use, etc. [9-10]. Studies on immaterial spatial elements show that cultural, inclusive and security of space have a significant positive impact on traditional public space [10-11]. Cultural resources and appropriate commercial forms of historical blocks also play an important role in enhancing the block vitality [12]. For the research on upgrading and transformation, scholars generally put forward strategies based on specific cases in combination with the conclusions of impact factor analysis [13-14]. Associated with data collection and research methods, traditional empirical observation and classified record analysis were mainly used in the early stage [15]. Recently, the development of big data has increased new data acquisition channels such as Baidu thermodynamic diagram, network POI data, mobile phone signaling [16].
\end{abstract} strategy. From the perspective of evaluation system, it 
In this context, there are abundant research perspectives on historical districts at present, while relatively little consideration is given to people's psychological needs and feelings in the selection of measurement indicators. Therefore, this study added the corresponding indicators that could reflect the subjective feelings of the population from the perspective of individual psychology. In data acquisition, observation, questionnaire survey and network open source data were combined to form more accurate research conclusions.

\section{RESEARCH AREA AND DATA SOURCE}

\subsection{Overview of the research area}

The research area includes the Songcheng historical district extending outwards from the ancient city wall. Up to now, it still retains some historical streets and buildings with unique Lingnan characteristics. It is a well preserved and representative ancient city wall historical block in Guangdong Province. The scope of this study extends to Nanxi road in the south, Songcheng road in the north, Kangle South Road in the West and Haoju road in the East. According to the map POI, it is supplemented and proofread in combination with the actual investigation, and finally it is obtained that there are 143 POI points in the ancient city wall historical block of Songcheng first road. Thus, the proportion of commercial facilities is much higher than that of other types, representing the dominant function of the block (as shown in Fig. 2)

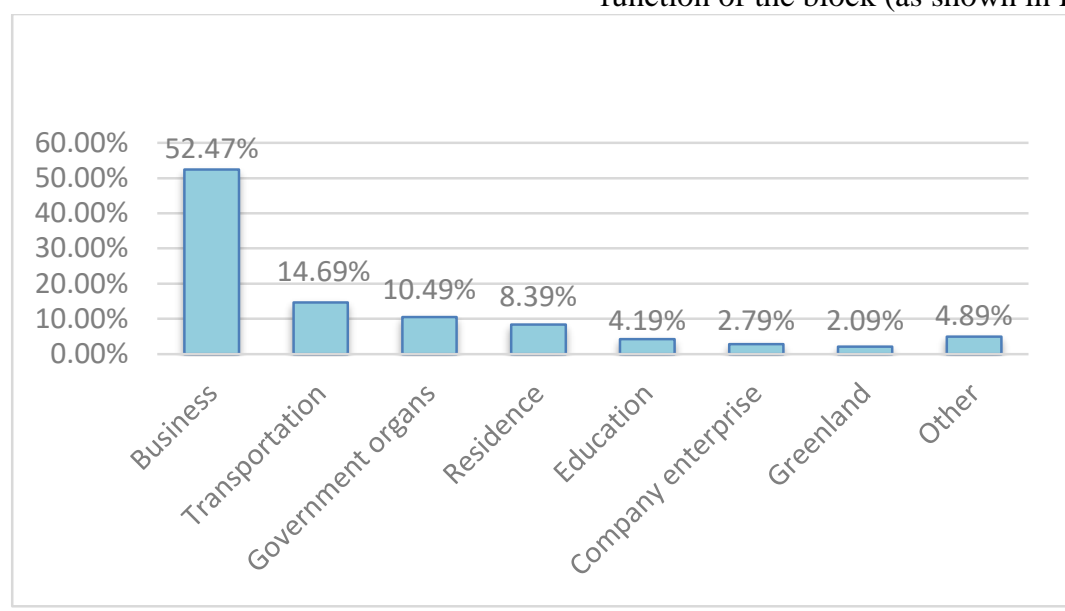

Figure 1. POI ratio of facilities in the research area

\subsection{Data sources}

The data of this study are mainly divided into the following three categories:

\subsubsection{Basic geographic data}

The high-definition electronic image of Songcheng first road historical block in Zhaoqing used in this study was downloaded through Baidu map. The road network of Songcheng first road historical block in Zhaoqing was downloaded through Open Street Map or Google Earth.

\subsubsection{Network data}

The POI data from Zhaoqing used in this study was collected by BIGEMAP software. POI points related to street vitality within $50 \mathrm{~m}$ on both sides of the street were selected according to the research area. Combined with the actual investigation, the collected data were supplemented and proofread. The obtained data included location, land type, name, etc. Baidu map was used to obtain the thermodynamic diagram data in different time periods of weekdays and holidays in Zhaoqing Songcheng first road historical block, thus analyzing the temporal and spatial distribution of block population.

\subsubsection{Field survey data}

In this study, a questionnaire survey and on-site observation were used to collect data for one and a half months to obtain the data of people's travel situation, activity time and activity range in historical districts. The research team randomly distributed 200 questionnaires and recovered 200, including 192 valid questionnaires, with an effective rate of $96 \%$. Alpha reliability coefficient method is often used to test the reliability of questionnaires. The value of $\alpha$ coefficient was 0.809 , indicating that the questionnaire had good reliability. Besides, bus stops, routes, 
public service facilities and other data were mainly observed through many field investigations and recorded accordingly.

Table 1. Data types and sources

\begin{tabular}{cccc}
\hline Data name & Format & Time & Sources \\
\hline $\begin{array}{c}\text { Regional scope of Songcheng first road historical } \\
\text { block in Zhaoqing }\end{array}$ & Vector & 2018 & Baidu map \\
$\begin{array}{c}\text { Road network of Songcheng first road historical block } \\
\text { in Zhaoqing }\end{array}$ & Vector & 2018 & $\begin{array}{c}\text { Open Street Map/Google } \\
\text { Earth }\end{array}$ \\
$\begin{array}{c}\text { POI data from Zhaoqing } \\
\text { Bus stops and routes }\end{array}$ & Excel & 2019 & BIGEMAP \\
$\begin{array}{c}\text { Excel } \\
\text { image }\end{array}$ & 2019 & Field survey \\
$\begin{array}{c}\text { Baidu thermal map } \\
\text { Ghaoqing high definition satellite remote sensing }\end{array}$ & Grid & 2018 & BIGEMAP \\
Scores on people's travel intention and historical blocks & Excel & 2020 & Questionnaire statistics \\
\hline
\end{tabular}

\section{RESEARCH METHODS}

three aspects: material elements, spatial scale and aesthetic attributes [17]. In the selection of indicators, the research results of block vitality evaluation indicators were taken as the basis [18]. Cultural facilities, cultural activities, activity experience, architectural features, visual effects, etc., were incorporated into the evaluation system based on field research and comprehensive consideration of people's psychological perception and block characteristics, as follows: find out the essential structure of multivariate observation variables and deal with dimension reduction. Referring to the classification of block evaluation factors by Chen et al, quantitative analysis of block vitality was carried out from

Table 2. Classification of block evaluation factor

\begin{tabular}{|c|c|c|c|}
\hline Serial numbers & Evaluation level & Main purpose & Evaluation factors \\
\hline 1 & \multirow{5}{*}{$\begin{array}{l}\text { Material element } \\
\text { level }\end{array}$} & \multirow{5}{*}{$\begin{array}{l}\text { Measure people's flow, } \\
\text { experience and material needs on } \\
\text { the street }\end{array}$} & A1 people's flow \\
\hline 2 & & & A2 cultural facilities \\
\hline 3 & & & A3 public service facilities \\
\hline 4 & & & A4 functionality \\
\hline 5 & & & A5 cultural activities \\
\hline 6 & \multirow{6}{*}{ Spatial scale level } & \multirow{6}{*}{$\begin{array}{l}\text { Reflect the external } \\
\text { environment of the individual } \\
\text { and the appropriate scale in the } \\
\text { block }\end{array}$} & B1 block location \\
\hline 7 & & & B2 block safety \\
\hline 8 & & & B3 spatial structure \\
\hline 9 & & & B4 overall layout \\
\hline 10 & & & B5 block density \\
\hline 11 & & & B6 traffic conditions \\
\hline 12 & \multirow{6}{*}{$\begin{array}{c}\text { Aesthetic attribute } \\
\text { level }\end{array}$} & \multirow{6}{*}{$\begin{array}{l}\text { Reflect the environmental } \\
\text { conditions and fun enjoyed by } \\
\text { individuals in the block }\end{array}$} & C1 greening coverage \\
\hline 13 & & & C2 block environment \\
\hline 14 & & & C3 block sanitation \\
\hline 15 & & & C4 architectural features \\
\hline 16 & & & C5 visual effects \\
\hline 17 & & & C6 activity experience \\
\hline
\end{tabular}

Based on the temporal variation law and spatial distribution characteristics in the block, the exploratory factor analysis and SPSS statistical method were used to test the 17 factors affecting the historical block vitality in the Songcheng wall by KMO and Bartlett spherical test. The data analysis results showed that KMO was 0.940 (> $0.6)$. The data passed Bartlett spherical test $(\mathrm{P}<0.05)$, meeting the preconditions of factor analysis and indicating that the data are quite suitable for further research.

\section{2. $A H P$}

AHP was used to analyze 17 indicators such as people's flow, cultural facilities, etc. By constructing 17 judgment matrices, the average value in each analysis item was calculated, and the judgment matrix was obtained by dividing its average value. By analyzing the weight value 
changes greatly during holidays. There will be three high peaks in historical blocks during holidays. The periods with high people's flow are morning, afternoon and evening respectively, and the highest peak of people's flow is higher than that on weekdays. During holidays, employment, school and other activities are reduced, and part of the vitality centers formed on weekdays will disperse or even disappear. Moreover, residents have more time and interest to go to historical blocks for activities during holidays, and the scope of activities in space is wider, thus forming multiple vitality centers.

\subsubsection{The block space has a single function and the crowd activity is short}

According to the questionnaire results, the residence time of people in historical blocks is mainly distributed in the range of 0.5-1.5 hours. Combined with the stage with dense activity population in the historical block, there will be an instantaneous thermal extreme value lasting 0.5-1.5 hours in the afternoon, evening and evening (i.e., the peak of people gathering activities). Based on the interview records, the short stay time is mainly due to the fact that most tourists think this is only a place for daily walking and leisure. The content of leisure facilities in the block is single, lacking characteristic activities and tourism products. At present, the only few arcade commercial contents are also relatively single and lack of attraction, and thus they are not regarded as weighty tourist attractions.

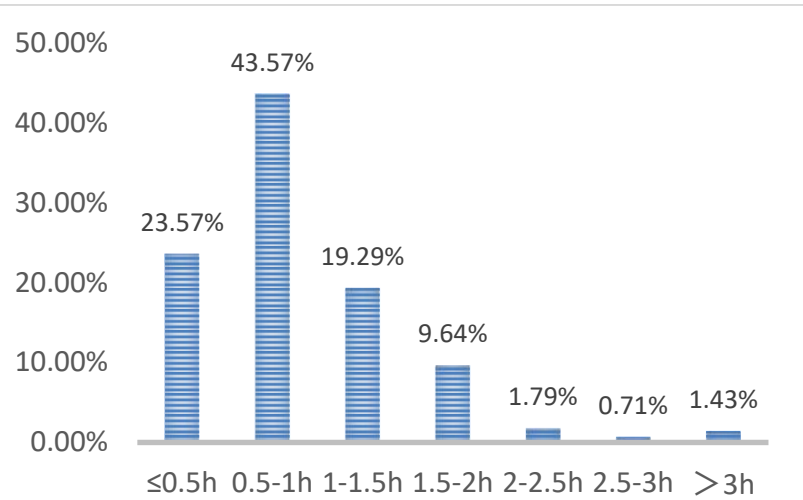

Figure 2. Population stay time in historical blocks

\subsubsection{Places with cultural characteristics and green space are favored, with diversified forms of crowd activities}

People's activities in Songcheng wall historical block are mainly concentrated in the green space on and under the city wall, the open space near the city wall, arcade commercial pedestrian street and ancient streets and lanes. Visitors are mostly distributed on the city wall, green space and ancient streets near the city wall. Leisure walkers are mainly distributed on the city wall, the green space under the city wall and the open space near the city wall. People pay more attention to the green environment and characteristic buildings in the selection standard of activity space.

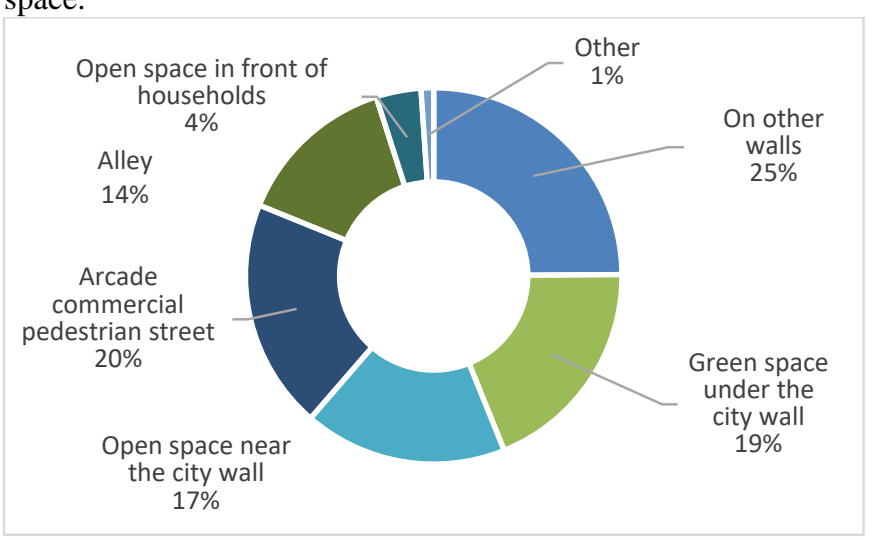

Figure 3. Distribution area of people's activities

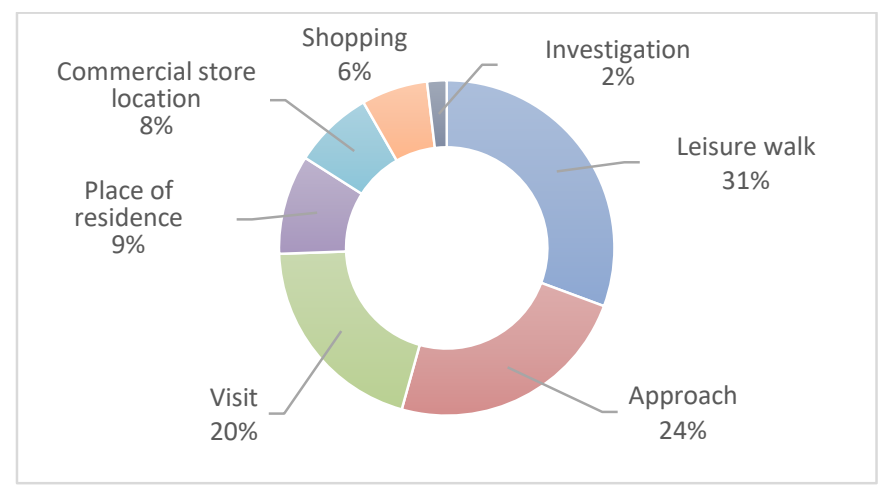

Figure 4. Form of people's activities

Through the questionnaire survey, it is found that the types and purposes of people's activities in the block are diverse. $58.21 \%$ of people's activities in the block are mainly leisure walking. $45 \%$ of people mainly commute through the block. $38.21 \%$ of people are mainly sightseeing, which is consistent with the network data analysis results. 
Table 4. Temporal and spatial distribution table of people's activities in the block

\begin{tabular}{|c|c|c|}
\hline Time interval & Spatial distribution & Main activity types \\
\hline $5: 30-7: 30$ am & Green space under sidewalk and city wall & Cleaning, walking and exercise \\
\hline 7:30-11:30 am & $\begin{array}{l}\text { City wall, green space under the city wall, arcade } \\
\text { commercial pedestrian block and historical block }\end{array}$ & $\begin{array}{l}\text { Commuting, sightseeing, shopping and } \\
\text { investigation }\end{array}$ \\
\hline $11: 30-13: 30$ noon & Sidewalk & Commuting \\
\hline $13: 30-17: 30 \mathrm{pm}$ & $\begin{array}{l}\text { Green space on and under the city wall, arcade } \\
\text { commercial pedestrian block and historical block }\end{array}$ & $\begin{array}{l}\text { Leisure walking, sightseeing, } \\
\text { shopping and investigation }\end{array}$ \\
\hline $17: 30-19: 30 \mathrm{pm}$ & $\begin{array}{l}\text { Green space under the city wall, open space near } \\
\text { the city wall and sidewalk }\end{array}$ & Commuting and leisure walking \\
\hline $19: 30-24: 00 \mathrm{pm}$ & $\begin{array}{l}\text { Green space under the city wall, open space near } \\
\text { the city wall, historical block and city wall }\end{array}$ & $\begin{array}{c}\text { Leisure walking, physical exercise and } \\
\text { visiting }\end{array}$ \\
\hline
\end{tabular}

\subsubsection{The activity group is mainly young people, and the space needs of different age groups are different}

According to the questionnaire survey, the visiting youth (aged 21-30) accounted for 52.14\%, accounting for more than half of the activity groups in the block. There is also a big gap in preference for space among different age groups. Young people prefer to go to the city wall scenic area, ancient streets and lanes, and arcade commercial pedestrian street. The middle-aged people mostly commute to bus stops and other point spaces on weekdays, and go to the city wall scenic spot and ancient streets for family activities on holidays. In addition to going to the ancient streets and alleys on holidays, the elderly groups mostly take leisure walks in the open space near the city wall, arcade commercial pedestrian street and the city wall.

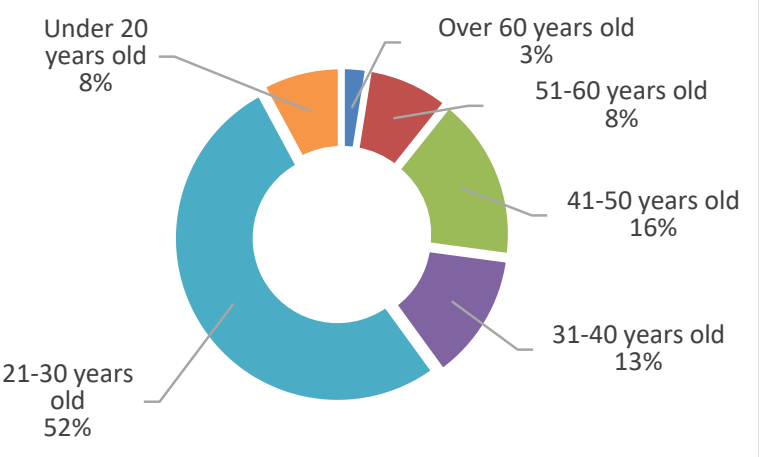

Figure 5. Age distribution of active population

\subsection{Historical block vitality evaluation status}

According to the factor score evaluation method, the factor evaluation scores of material elements, spatial scale and aesthetic attributes can be calculated based on the questionnaire survey results.

\subsubsection{Material elements}

The average evaluation score of people's flow, cultural facilities, public service facilities, functionality and cultural activities was 4.83 . The public service facilities and functional factors are lower than the average score. It can be seen that most people think that the public service facilities and functionality of ancient city wall historical block in Songcheng first road are poor (i.e., their satisfaction with this is low).

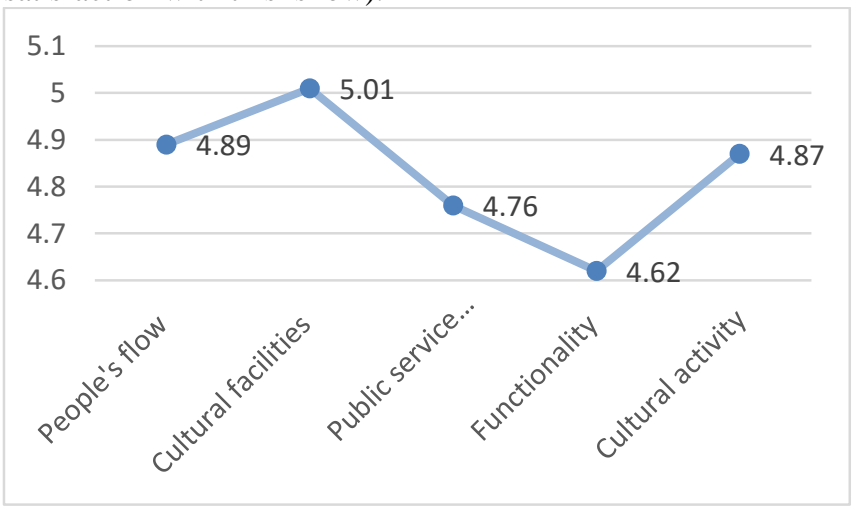

Figure 6. Factor evaluation score at material elements

\subsubsection{Spatial scale}

The average score of block location, block safety, spatial structure, overall layout, block density and traffic condition at the spatial scale level was 5.105. Among them, the block density is low, accounting for 4.85 points. This reflects that the external environment perceived by the crowd in the ancient city wall historical block of Songcheng first road is more appropriate, while the internal scale of the block still needs to be improved. 


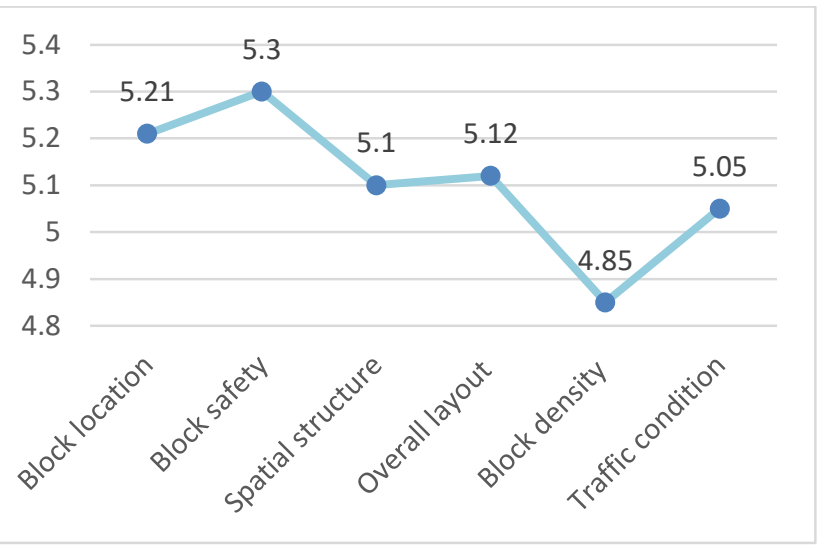

Figure 7. Factor evaluation score at spatial scale

\subsubsection{Aesthetic attributes}

At the aesthetic attribute level, the evaluation scores of greening coverage, block environment, block health, architectural features, visual effects and activity experience were higher than 5, reflecting the good environmental conditions and high degree of fun enjoyed by individuals in the block. At the same time, it can be seen that the activity group has a high degree on recognition for the six factors at the aesthetic attribute level, and the architectural features score is the highest at the same level, followed by visual effects.

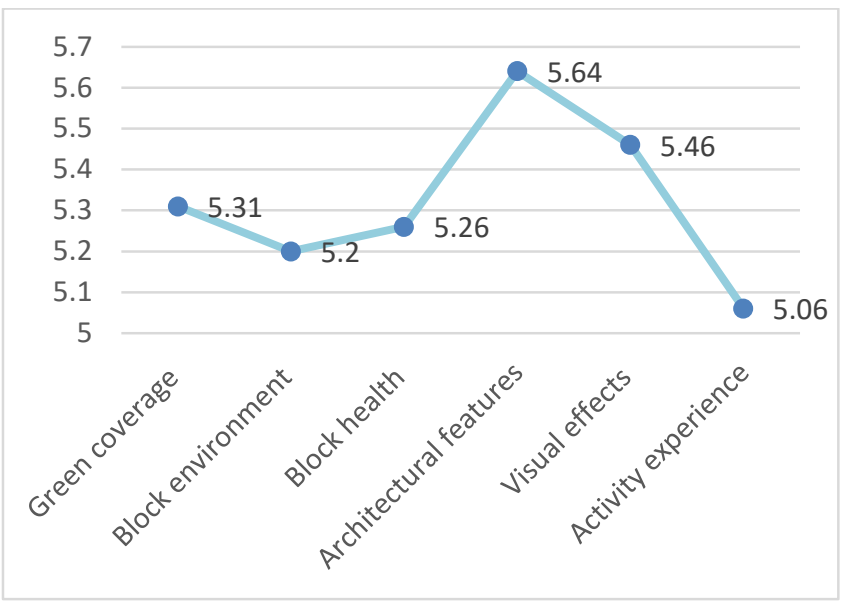

Figure 8. Factor evaluation score at aesthetic attribute

\subsection{Influencing factors of historical block vitality}

In this context, SPSS statistical software was used to model and the maximum variance rotation method was used to find the relationship between the factors and the corresponding research level. Table 6 shows the factor load coefficient after rotation, which is the information extraction of corresponding factors at the three research levels and the relationship between these factors and the three research levels

Table 5. Factor load coefficient after rotation

\begin{tabular}{|c|c|c|c|c|}
\hline \multirow{2}{*}{ Name } & \multicolumn{3}{|c|}{ Factor load coefficient $\square$} & \multirow{2}{*}{$\begin{array}{c}\text { Common degree (common } \\
\text { factor variance) }\end{array}$} \\
\hline & Factor 1 & Factor 2 & Factor 3 & \\
\hline A1 people's flow & 0.367 & 0.591 & 0.058 & 0.487 \\
\hline A 2 cultural facilities & 0.223 & 0.781 & 0.290 & 0.744 \\
\hline A3 public service facilities & 0.233 & 0.790 & 0.267 & 0.750 \\
\hline A4 functionality & 0.287 & 0.798 & 0.162 & 0.745 \\
\hline A5 cultural activities & 0.190 & 0.648 & 0.402 & 0.618 \\
\hline B1 block location & 0.734 & 0.276 & 0.145 & 0.636 \\
\hline B2 block safety & 0.715 & 0.214 & 0.258 & 0.623 \\
\hline B3 spatial structure & 0.719 & 0.231 & 0.408 & 0.737 \\
\hline B4 overall layout & 0.595 & 0.407 & 0.364 & 0.652 \\
\hline B5 block density & 0.728 & 0.311 & 0.248 & 0.687 \\
\hline B6 traffic conditions & 0.654 & 0.263 & 0.331 & 0.607 \\
\hline $\mathrm{C} 1$ greening coverage & 0.521 & 0.235 & 0.573 & 0.655 \\
\hline $\mathrm{C} 2$ block environment & 0.517 & 0.194 & 0.682 & 0.769 \\
\hline C3 block sanitation & 0.503 & 0.198 & 0.635 & 0.696 \\
\hline $\mathrm{C} 4$ architectural features & 0.240 & 0.278 & 0.806 & 0.784 \\
\hline C5 visual effects & 0.240 & 0.306 & 0.807 & 0.802 \\
\hline C6 activity experience & 0.261 & 0.573 & 0.565 & 0.715 \\
\hline
\end{tabular}

Note: Bold numbers indicate that the absolute value of load factor is greater than 0.4.

It can be seen from the above table that the common values of corresponding factors at all research levels are higher than 0.4, indicating that there is a strong correlation between the three levels of spatial scale (factor 1), material elements (factor 2) and aesthetic attributes (factor 3) and their factors. Three factors were extracted by exploratory factor analysis. The cumulative variance explanation rate of the three factors after rotation was $68.881 \%$. It is calculated that the material element level accounts for $32.27 \%$, and the spatial scale level accounts for $35.96 \%$, 
and the aesthetic attribute level accounts for $31.77 \%$. The proportion of factors in the three levels is relatively average and has strong correlation.

Table 6. Variance interpretation rate

\begin{tabular}{|c|c|c|c|c|c|c|c|c|c|}
\hline \multirow[b]{2}{*}{$\begin{array}{l}\text { Factor } \\
\text { number }\end{array}$} & \multirow[b]{2}{*}{$\begin{array}{l}\text { Latent } \\
\text { root }\end{array}$} & \multicolumn{2}{|l|}{ Latent root } & \multicolumn{3}{|c|}{$\begin{array}{c}\text { Variance interpretation rate } \\
\text { before rotation }\end{array}$} & \multicolumn{3}{|c|}{$\begin{array}{c}\text { Variance interpretation rate after } \\
\text { rotation }\end{array}$} \\
\hline & & $\begin{array}{c}\text { Variance } \\
\text { interpretatio } \\
\text { n rate } \%\end{array}$ & $\begin{array}{l}\text { Accumulati } \\
\text { on } \% \square\end{array}$ & $\begin{array}{l}\text { Latent } \\
\text { root }\end{array}$ & $\begin{array}{c}\text { Variance } \\
\text { interpretati } \\
\text { on rate } \%\end{array}$ & $\begin{array}{l}\text { Accumulatio } \\
\text { n } \% \square\end{array}$ & $\begin{array}{l}\text { Latent } \\
\text { root }\end{array}$ & $\begin{array}{c}\text { Variance } \\
\text { interpretation } \\
\text { rate } \%\end{array}$ & $\begin{array}{l}\text { Accumulat } \\
\text { ion } \%\end{array}$ \\
\hline & 9.405 & 55.321 & 55.321 & 9.405 & 55.321 & 55.321 & 4.211 & 24.773 & 24.773 \\
\hline 2 & 1.328 & 7.811 & 63.132 & 1.328 & 7.811 & 63.132 & 3.778 & 22.225 & 46.997 \\
\hline 3 & 0.977 & 5.750 & 68.881 & 0.977 & 5.750 & 68.881 & 3.720 & 21.884 & 68.881 \\
\hline 4 & 0.823 & 4.840 & 73.721 & - & - & - & - & - & - \\
\hline 5 & 0.692 & 4.070 & 77.791 & - & - & - & - & - & - \\
\hline 6 & 0.541 & 3.184 & 80.974 & - & - & - & - & - & - \\
\hline 7 & 0.445 & 2.620 & 83.594 & - & - & - & - & - & - \\
\hline 8 & 0.425 & 2.502 & 86.096 & - & - & - & - & - & - \\
\hline 9 & 0.362 & 2.130 & 88.226 & - & - & - & - & - & - \\
\hline 10 & 0.358 & 2.108 & 90.334 & - & - & - & - & - & - \\
\hline 11 & 0.301 & 1.773 & 92.107 & - & - & - & - & - & - \\
\hline 12 & 0.292 & 1.719 & 93.827 & - & - & - & - & - & - \\
\hline 13 & 0.263 & 1.548 & 95.375 & - & - & - & - & - & - \\
\hline 14 & 0.239 & 1.407 & 96.782 & - & - & - & - & - & - \\
\hline 15 & 0.212 & 1.245 & 98.027 & - & - & - & - & - & - \\
\hline 16 & 0.177 & 1.042 & 99.069 & - & - & - & - & - & - \\
\hline 17 & 0.158 & 0.931 & 100.000 & - & - & - & - & - & - \\
\hline \multicolumn{10}{|c|}{$\begin{array}{l}\text { The weight value and eigenvector value of } 17 \text { influencing } \\
\text { factors were obtained by AHP. The results showed that the } \\
\text { weight of people's flow, cultural facilities, public service } \\
\text { facilities, functionality, cultural activities, traffic } \\
\text { conditions and activity experience was } 5.882 \% \text { lower than } \\
\text { the average weight value. Among them, public service }\end{array}$} \\
\hline
\end{tabular}

Table 7. AHP analysis results

\begin{tabular}{|c|c|c|c|c|}
\hline Impact Factor & Eigenvector & Weight value & Maximum eigenvalue & CI value \\
\hline A1 people's flow & 0.959 & $5.640 \%$ & & \\
\hline A2 cultural facilities & 0.983 & $5.780 \%$ & & \\
\hline A3 public service facilities & 0.934 & $5.491 \%$ & & \\
\hline A4 functionality & 0.906 & $5.331 \%$ & & \\
\hline A5 cultural activities & 0.955 & $5.619 \%$ & & \\
\hline B1 block location & 1.022 & $6.014 \%$ & & \\
\hline B2 block safety & 1.039 & $6.113 \%$ & & \\
\hline B3 spatial structure & 1.000 & $5.883 \%$ & & \\
\hline B4 overall layout & 1.004 & $5.903 \%$ & 17.000 & 0.000 \\
\hline B5 block density & 0.951 & $5.594 \%$ & & \\
\hline B6 traffic conditions & 0.990 & $5.821 \%$ & & \\
\hline $\mathrm{C} 1$ greening coverage & 1.041 & $6.122 \%$ & & \\
\hline C2 block environment & 1.019 & $5.994 \%$ & & \\
\hline C3 block sanitation & 1.032 & $6.068 \%$ & & \\
\hline $\mathrm{C} 4$ architectural features & 1.105 & $6.501 \%$ & & \\
\hline C5 visual effects & 1.070 & $6.295 \%$ & & \\
\hline C6 activity experience & 0.992 & $5.833 \%$ & & \\
\hline
\end{tabular}


According to exploratory factor analysis and AHP, it can be seen that the historical block vitality mainly has three dimensions: material elements, spatial scale and aesthetic attributes. Among them, material elements express the basic functional attributes in historical blocks, and are the premise and basis of people's activities. Spatial scale is an important factor for people to feel comfortable in the process of activities. The aesthetic attributes consider the psychological perception of people's activities in the block. The weight of these three dimensions is average, and the proportion of aesthetic attributes is slightly higher, indicating that aesthetic attributes have a stronger correlation with the measurement of historical block vitality.

Besides, the strength distribution of factors can be seen from the block location, block safety at the spatial scale level and the greening coverage, block health, architectural characteristics and visual effect at the aesthetic attribute level. These six factors have a higher weight, which has a great influence on the vitality measurement of historical blocks in the Songcheng wall. On the contrary, the traffic flow, cultural facilities, public service facilities, functional and cultural activities at the material element level, the traffic conditions at the spatial scale level and the activity experience at the aesthetic attribute level have a low weight in the exploratory factor analysis, and have a weak correlation with the historical block vitality in the Songcheng wall.

\section{STRATEGIES FOR IMPROVING BLOCK VITALITY}

\subsection{Maintaining the block characteristics and improving the material space quality}

Through this study, it can be seen that most tourists have low satisfaction with the material elements of the historical blocks in the Songcheng wall, and the poor sanitation will greatly reduce the visual experience of the block. It should pay attention to the block sanitation, keep the street comfortable and tidy, reasonably set up garbage cans and centralized garbage disposal places. It should not only do a good job in the block sanitation, but also ensure that it conforms to the block characteristics and does not affect the beauty of people. Besides, characteristic buildings and block space scale are the main sources of people's impression on the block and play an important role in attracting vitality. On the one hand, the damaged or peeled exterior walls of historical block buildings should be repaired and unified architectural style, thus improving the facade aesthetics of block buildings and continue the vitality of the ancient city wall. On the other hand, it should try to design a reasonable and pleasant space scale of the block in the reconstruction, and avoid blindly creating large scale space and destroying the original block texture. Greening leisure space is also an important node for the historical block to attract people to stop and improve the sense of block quality. Integrating block space and improving greening quality can greatly improve the experience sense of the historical block. Therefore, the suitability of block space environment can be improved by means of waste space utilization and three-dimensional greening when transforming the spatial layout of historical blocks.

\subsection{Developing in-depth tourism projects and enhancing the block vitality quality}

The different characteristics vitality in space and regular time elements are organically combined by using the time changes and spatial differences of the historical block vitality. The peak value of instantaneous vitality and the quality of local spatial vitality are improved, and tourism projects that attract tourists in the depth of the block are developed. For example, temporary shops selling tourist souvenirs related to historic districts may be set up in areas with weak population vitality, or convenient snack stalls may be allowed. Special and innovative tourism projects in areas with large passenger flow should be added, thus revitalizing the local space lacking vitality and achieving the purpose of "staying" tourists.

\subsection{Enriching the block activities and enhancing people's cultural identity}

Historical block is an important carrier for the dissemination and development of urban history and culture. To create the block vitality, it can enrich the block and urban culture by organizing temporary cultural activities such as street culture exhibition, outdoor art exhibition or relevant historical and cultural competitions. It is necessary to pay attention to the needs of residents daily cultural activities, improve the layout of public cultural facilities in the block space, add relevant historical and cultural facilities in the block, and enhance the people's cultural identity and happiness.

Considering that the historical blocks in many cities are similar to the historical blocks in the Songcheng wall, which is not only a simple scenic spot, but also an area integrating tourism, urban residence and daily leisure functions. Users are also more diversified than general scenic spots. Therefore, more attention should be paid to the construction of public service facilities inside the blocks in the future maintenance and construction, such as reasonably adding corridors and kiosks for tourists, configuring public toilets, etc. Historical blocks should not only focus the development and improvement of commercial facilities, but also pay attention to the combination of street environment and local history to create an attractive historical block suitable for people gathering, bearing diverse activities. 


\section{CONCLUSION}

In this context, evaluation index system of spatial vitality in historical blocks was constructed from three aspects: material elements, spatial scale and aesthetic attributes. Exploratory factor analysis and AHP were used to measure and weight the vitality factors. Findings are as follows:

According to the questionnaires and interviews results, the public service facilities and functionality of the historical block in Songching wall are relatively poor at the material element level, and the people have low satisfaction with the public service facilities and functionality of the block. People have the highest satisfaction with the aesthetic attributes of the block, especially the architectural features, which are the core elements of the historical block in Songching wall to attract people.

Through the analysis on temporal and spatial behavior characteristics of the historical block vitality, it is found that the change range of the spatial vitality in historical block on holidays is larger than that on weekdays, and a more concentrated vitality center will be formed in the scenic spots. The main vitality peak hours are in the afternoon and evening, and the lack of in-depth sightseeing projects leads to a shorter duration for people's activities. People's activity forms in the block present diversified characteristics, while they do not bring the prosperity of commercial activities in the block. Block users are mainly young and old. People at different ages have different needs for blocks. The youth group is more inclined to leisure and recreation by themselves or with friends, and the middle-aged group is more likely to commute and play with their families, and the elderly group is more likely to take daily walks and exercise.

Through this study, it is found that there is a strong correlation between various factors of the evaluation index system and the historical block vitality, among which there are differences among the types of internal components. From the aspect of material factors, people's flow, cultural facilities, public service facilities, functions and cultural activities are the weak correlation factors of block vitality, which have little influence on block vitality. From the perspective of spatial scale, block location and block safety are strong correlation factors, and spatial structure, overall layout and block density are strong correlation factors, and traffic conditions are weak correlation factors. From the perspective of spatial scale, block location and block safety are strong correlation factors, and spatial structure, overall layout and block density are strong correlation factors, and traffic conditions are weak correlation factors. From the factor strength distribution, it can be seen that location and security are important indicators affecting the block vitality from the macro perspective of the block, followed by factors closer to the living scale of the population such as spatial structure, overall layout and block density. From the perspective of aesthetic attributes, green coverage, block sanitation, architectural features and visual effects are strongly related factors, and block environment is a strong related factor, and activity experience is a weak related factor. Through the factors strength, it can be found that the greening coverage, block health, architectural features and visual effects have an important impact on the block vitality. People have subjective intentional cognition on overall situation of the block. A good and comfortable environment is easier to stimulate people's activities, thus affecting the block vitality.

\section{ACKNOWLEDGMENT}

This work was supported by Guangdong provincial key platform for universities and youth innovative talents project (No. 2019WQNCX154) and provincial innovation and entrepreneurship training program for college students in 2019 (No. S201913902021).

\section{REFERENCES}

[1] Xue Kai, Yue Lizhong. Vitality Characteristics and Promotion Strategies of Public Space in Qingdao Dabao Island Historical Block [J]. Science, Technology and Engineering, 2020, 20 (21): 8757-8765.

[2] Mao Zhirui, Chen Xiaokui, Xiang Zhenhai, et al. Measurement and Influencing Factors of Block Vitality in Historical Block - Taking the Wenming Block in Kunming as an Example [J/OL]. Southern Architecture: 1-11 [2021-03-10].

[3] Ashihara Yoshinobu, Translated by Yin Peitong. Aesthetics of Streets [M]. Baihua Literature and Art Publishing House, 2006.

[4] Gharyan, Translated by He Renke. Communication and Space $[\mathrm{M}]$. China Construction Industry Press, 2002.

[5] Kevin Lynch, Translated by Fang Yiping. City Image [M]. Beijing: Huaxia Publishing House, 2001.

[6] Norberg Schultz. Place Spirit - Towards Architectural Phenomenology [M]. Huazhong University of Science and Technology Press, 2010.

[7] Han Le, Li Ni. Research on the Evaluation System of Urban Historical Blocks Based on the Public Space Vitality [J]. Natural Science Journal of Xiangtan University, 2014, 36 (03): 122-126.

[8] Li Jiajing. Discussion on the Planning and Protection Model of Historical Areas Based on Development "Vitality" [D]. Southeast University, 2015.

[9] Niu Xinyi, Wu Guanshu, Li Meng. Study on the Impact of Built Environment on Street Vitality and Its Temporal and Spatial Characteristics Based on LBS 
Positioning Data [J]. International Urban Planning, 2019, 34 (01): 28-37.

[10] Huang Dan, Dai Donghui. Influence of Life Street Elements on Vitality - Taking Typical Blocks in Shenzhen as an Example [J]. Chinese Garden, 2019, 35 (09): 89-94.

[11] Bao Yafang, Sun Zhi, Song Mengke, et al. Influencing Factors of Public Space Cultural Vitality in Traditional Villages in Lanxi, Zhejiang Province from the Perspective of Residents' Perception [J]. Regional Research and Development, 2019, 38 (05): 175-180.

[12] Zhang Yuyang, Yang Changming, Qi Ling. Evaluation and Influencing Factors of Street Vitality in Historical Blocks - Taking Shichahai Historical Block as an Example [J]. Chinese Garden, 2019, 35 (03): 106-111.

[13] Li Jingxiao. Research on the Revitalization of Public Space in Historical Blocks Based on Traditional Residential Culture [D]. Xi'an University of Architecture and Technology, 2011.
[14] Zhang Yibing, Han Zilong, Feng He, et al. Research on Progressive Renewal in Historical and Cultural Blocks from the Perspective of Block Vitality Taking Wanzi Street Historical Block in Yangzhou City as an Example [J]. Architecture and Culture, 2017 (07): 230-232.

[15] Min Zhongrong, Ding Fan. Analysis on Temporal and Spatial Distribution Characteristics of Street Vitality Based on Baidu Thermal Map - Taking the Historical Urban Area of Nanchang, Jiangxi Province as an Example [J]. Urban Development Research, 2020, 27 (02): 31-36.

[16] Xu Yangfei, Zheng Siqi, Wang Jianghao. Urban Vitality: Demand and Supply of Localized Consumption Opportunities [J]. New Architecture, 2016 (01): 26-31.

[17] Long Ying, Zhou Yin. Quantitative Evaluation and Influencing Factors of Block Vitality - Taking Chengdu as an Example [J]. New Architecture, 2016 (01): 52-57.

[18] Zhang Ying. Measurement and Impact Mechanism of Urban Block Vitality [D]. Wuhan University, 2019. 